\title{
Effects of flash luminance and positional expectancies on visual response latency
}

\author{
HOWARD C. HUGHES \\ Dartmouth College, Hanover, New Hampshire
}

\begin{abstract}
It is well established that human observers respond more quickly to visual targets that appear in expected locations than they do to ones in unexpected locations. These variations in simple reaction time have been attributed to a covert alignment of an attentional mechanism to the expected target location. The present experiments investigated the influence of strength of signal and strength of subject's positional expectancy on the magnitude of this attentional effect. In the first experiment, target luminance was varied over a range of three log units, and it was found that the effects of luminance were essentially additive with the effect of the positional expectancy (i.e., the attention effect). The second experiment found that the magnitude of visual attention interacts with the information value of the precue used to create the spatial expectancy, although, once again, luminance had additive effects. The resuls are interpreted as indicating that, rather than influencing early visual processing, the act of attending to a spatial location operates fairly late in the detection process.
\end{abstract}

It is well documented that observers can process visual inputs more effectively when they have prior information about where the target is likely to occur (e.g., Eriksen \& Collins, 1969; Eriksen \& Hoffman, 1972). The facilitatory effect of this prior information is generally attributed to the observer's ability to direct his or her attention to the expected source of input (Colegate, Hoffman, \& Eriksen, 1973). While the increase in processing efficiency seen for expected locations is firmly established, the locus of these attentional influences is less clear. Some evidence has indicated that attention serves to enhance early visual processing (e.g., Eriksen \& Hoffman, 1974; Posner, Snyder, \& Davidson, 1980), while other results have been used to argue for late selection (Eriksen \& Hoffman, 1973; Eriksen \& Spencer, 1969; Shiffrin \& Gardner, 1972; Shiffrin, Gardner, \& Allmeyer, 1973; Skelton \& Eriksen, 1976).

Posner and his colleagues (e.g., Posner, 1978; Posner et al., 1980) have developed a paradigm that permits measurement of both the benefits and the costs associated with directing attention to an eccentric location. Using the cost-benefit analysis, they have shown that in a simple reaction time (RT) task, observers respond more quickly to targets that occur in expected locations and respond more slowly to targets occurring in unexpected locations. RTs on non-

This research was supported in part by a faculty research award from Dartmouth College. I wish to thank R. Brown and D. Traweek for assistance in conducting these experiments, and J. C. Baird, C. A. Fowler, G. L. Wolford, L. D. Zimba, and W. M. Layton for critical readings of the manuscript.

The author's mailing address is: Department of Psychology, Dartmouth College, Hanover, NH 03755. informative (neutral) trials are at intermediate levels. These differences in RT have also been interpreted in terms of the alignment of attention with the expected input location. Posner et al. (1980) suggest that both the costs and the benefits can be accounted for in terms of an interaction of central attentional effects with early visual processing. They propose a distinction between detection (analogous in most respects to conscious detection) and orienting, which they define as aligning sensory and/or central attentional systems with a specific input channel (or location). By making this distinction, Posner et al. (1980) suggest the possibility that orienting can precede detection. It is through this suggestion that they account for the costs and benefits of directed visual attention: the benefits derive from an early enhancement process, whereas the costs derive from the necessity of moving (reorienting) attention from the expected to the unexpected location (on invalid trials) prior to detection. Thus, Posner et al. (1980) suggest (1) that attention is a spatially focal, unitary process, (2) that it can be moved throughout the visual field, but that, at any given moment, it has only one focus (positions are selected serially; e.g., Shulman, Remington, \& McLean, 1979), and (3) that attention acts to enhance early visual processing.

The present paper is primarily concerned with the issue of early visual enhancement. The additive-factors approach (Sternberg, 1969) was applied to two variables that might be expected to influence RTs in this paradigm and therefore to provide some insight into the issue of early versus late attentional selection. The first experiment explored the role of signal intensity on the relative costs and benefits of attending to an eccentric spatial location, and the second 
experiment examined the effects of the information value of the spatial precue used to induce shifts in attention.

\section{EXPERIMENT 1}

Interest in the role of signal strength derives from the suggestion (Posner et al., 1980) that the attentional mechanism might serve to enhance the early processing of visual information, thereby producing a facilitatory effect on detection latency. Thus, one might envision a mechanism that could increase signal-to-noise ratios or otherwise enhance the signals very early in visual processing. Relevant anatomical studies of the central visual pathways have revealed connections between a variety of so-called nonspecific areas within the brainstem reticular formation and the lateral geniculate nucleus and the visual cortex (for references, see Hughes, 1980, and Hughes \& Mullikin, 1984). These reticular areas have long been thought to mediate changes in attention and alertness, and physiological studies of these pathways have indicated that these reticular effects are essentially facilitatory (more precisely, disinhibitory) on neurons in the geniculocortical projection system (for a review, see Singer, 1977). One can imagine that attentional enhancement could operate through such a reticular system, and thus facilitate visual processing at an early stage. The rationale of the present experiment is that, under such circumstances, one might expect to find evidence that the magnitude of the attention effect varies with signal strength. Specifically, the expectation is that the effect would diminish with increasing target intensity, as any enhancement of strong signals would lead to little improvement in detection latency because the responses of these visual neurons would begin to approach the saturation level of the system's response capability. Simply put, early enhancement suggests that attention should be more beneficial when the target is difficult to detect.

The experiment also addresses additive-factors logic (Sternberg, 1969), in which an interaction between attentional enhancement and signal intensity would suggest that both attention and luminance operate at the same (early) processing stage, whereas additivity would suggest that attention operates later in signal processing.

\section{Method}

The stimulus display. A computer-controlled video raster monitor was used to create the display, which consisted of a fixation point, a precue, and a visual target that could occur to the left or right of fixation. A left- or right-pointing arrow served as the precue, and was located adjacent to the fixation mark. The entire display was $16.6 \mathrm{deg}$ wide and $14.2 \mathrm{deg}$ high. The fixation mark and the precues were $0.5 \times 0.5 \mathrm{deg}$, and the target subtended $0.033 \mathrm{deg}$ of arc. The targets appeared on a black background 4.0 deg on either side of fixation. The viewing distance was $67 \mathrm{~cm}$.
To vary target luminance, the display was viewed through neutral density filters, with luminance being varied in one-log-unit steps from 6.0 to $0.006 \mathrm{mfL}$. The duration of the flash was $100 \mathrm{msec}$ (onset/offset time of $16 \mathrm{msec}$ ). A schematic illustration of the display is shown in Figure 1. The apparatus was located in an isolated, completely darkened room.

The task. The task was a simple RT to the occurrence of the target. The subjects responded to the flashes by depressing a microswitch as quickly as possible, using the finger and hand that seemed most comfortable to them. The microswitch was polled each millisecond, so the RTs are accurate to the nearest millisecond. Eye position was monitored with bitemporally placed dc (Ag-AgCl, Beckman Instruments) recording electrodes. The signals from these electrodes were digitized (sampling rate of $100 \mathrm{~Hz}$ ) after high-gain differential amplification. The electrooculogram (EOG) used had a sensitivity of about $1.0 \mathrm{deg}$, and deviations from fixation that exceeded $1.0 \mathrm{deg}$ prior to flash onset automatically reset the trial. The EOG was calibrated as follows. The fixation mark was presented, and the subjects were instructed to fixate the spot steadily until it went off (about $3 \mathrm{sec}$ ). While the subject fixated this spot, the computer took analog-to-digital conversions from the electrodes, and the range of digital values was used to create a fixation "window." The same procedure was used for the two target locations (on either side of fixation). If the range of the fixation window did not overlap with similar windows for the two target locations, and the overall range was within previously established values for good fixation, then the experimenter continued with the session. The calibration procedure preceded each run of the program (each run lasted about $20 \mathrm{~min}$ ) and two runs of the program completed an experimental session (see procedure section).

Procedure. The procedure was similar to that used by Posner et al. (1980). There were three types of trials, designated valid, invalid, and neutral. On valid trials, the target appeared on the side indicated by the arrow precue; on invalid trials, the target appeared on the side opposite to that indicated by the arrow. On neutral trials, both a left- and a right-pointing arrow were presented, indicating that the target could occur with equal probability on the left or right of fixation. There were $14.3 \%$ neutral trials; of the remaining trials, $83.3 \%$ were valid and $16.7 \%$ were invalid. The

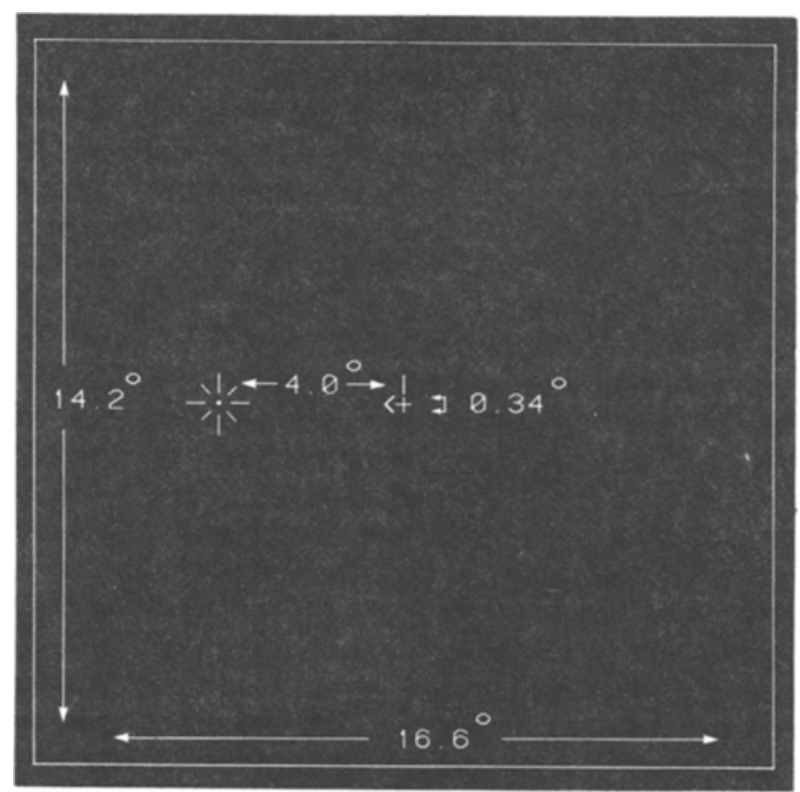

Figure 1. A schematic illustration of the stimulus display. The nash is indicated by the point with radial lines. 
interval between the onset of the precue and the target onset (SOA) varied randomly between 1,000 and $2,000 \mathrm{msec}$. These relatively long SOAs were used because previous work had shown that the beneficial effects of the precue asymptote at $250-300 \mathrm{msec}$ (Posner, 1980; Tsal, 1983), and I wanted to be sure that the attentional mechanism had arrived at the expected location on all trials. The precue remained on until the subject responded. A warning tone $(1000 \mathrm{~Hz}, 250 \mathrm{msec})$ was presented simultaneously with the onset of the precue. Each subject participated in one practice session (332 trials) and four experimental sessions (one per day), with each session lasting approximately $1 \mathrm{~h}$. Signal intensity varied across sessions according to a Latin square. The subjects were always informed about the accuracy of the precue $(83 \%$ accurate in this experiment). They were instructed to use the information provided by the arrow to their advantage by trying to attend to the cued location, with the constraint that they not move their eyes. They were told that if they did break fixation, the screen (with the exception of the fixation mark) would go blank, and the trial would start over.

Subjects. Eight undergraduate students served as subjects. For their participation, they received course credit in an introductory psychology course. All subjects either claimed to be emmetropic or were optically corrected.

\section{Results}

Anticipatory error rates. All responses that either preceded the flash or occurred within $100 \mathrm{msec}$ of flash onset were considered anticipatory and were excluded from the principal data analysis. These anticipations were analyzed separately. Anticipation rates were low: $3.3 \%$ on valid trials, $3.17 \%$ on neutral trials, and $3.3 \%$ on invalid trials. An analysis of variance on these rates (intensity $\times$ cue type $\times$ subjects) failed to reveal any significant differences in the rates of anticipation errors.

Reaction time data. Figure 2 shows the mean latency for valid, invalid, and neutral trials at each of the four flash intensities. It is apparent that the predicted increase in the costs and benefits of directed

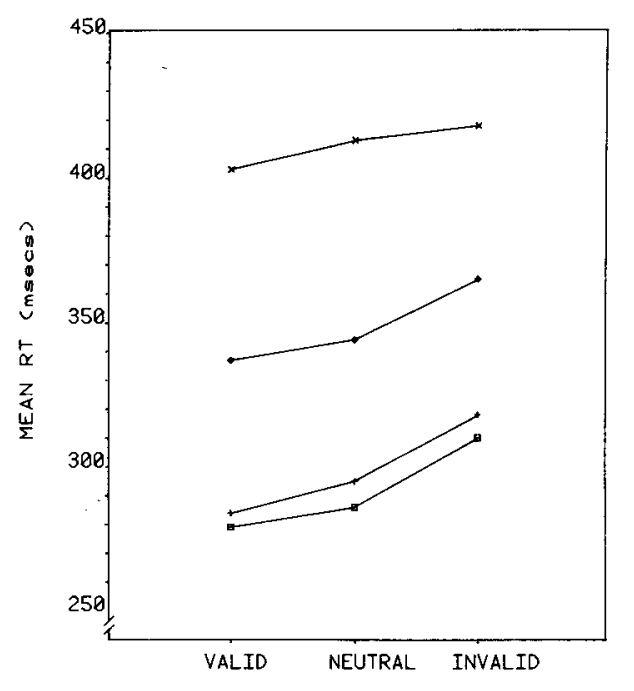

Figure 2. Effects of flash luminance on visual RTs on valid, neutral, and invalid trials. Each curve represents data from a different luminance. Squares represent the brightest flashes, and $x s$ represent the dimmest flashes. Specific luminance values are given in the text. visual attention with decreasing flash intensity was not obtained. Analysis of variance on the individual subject means revealed significant intensity $[F(3,21)=$ 53.3] and cuing effects $[F(2,14)=47.7$; both ps $<.001]$ and a significant cuing $x$ intensity interaction $[F(6,42)=2.69, p<.03]$. However, it is clear that the cuing $X$ intensity interaction is not in the predicted direction (the effect became smaller with decreasing flash intensity rather than larger). Pairwise comparisons, using the Newman-Keuls procedure, indicated that valid trials were significantly $(p<.05)$ faster than invalid trials at all four intensities. Invalid trials were significantly slower than neutral trials for all but the lowest flash luminance, whereas valid trials were faster than neutral trials in two of the four comparisons (the second brightest and the dimmest flash luminances).

\section{Discussion}

The results indicate that the effects of directed visual attention are essentially additive with flash intensity. Although there was an interaction between the size of the attention effect and signal strength, the direction of this interaction was opposite to that expected on the assumption that attention enhances early visual processing. I would tend to attribute this interaction to a ceiling effect on the invalid trials, which reflects the difficulty in moving simple RTs to brief flashes beyond about $420 \mathrm{msec}$ (see Hughes \& Kelsey, 1984).

In discussing the issue of early versus late enhancement, it is important to specify exactly what is meant by the terms "early" and "late." Many authors (e.g., Eriksen \& Hoffman, 1973; Shiffrin et al., 1973) regard "late" selection in terms of the processes assumed to precede the attentional effects (e.g., sensory encoding, detection, short-term memory, etc.). "Early" typically corresponds to events prior to detection (e.g., Posner, 1978; Posner et al., 1980; Shiffrin et al., 1973).

The additivity of the effects of attention and signal strength may be interpreted as evidence that directed visual attention and signal strength operate at different stages of the signal detection process (cf. Sternberg, 1969). Since luminance influences the earliest stages of visual processing, it seems likely that attention operates at some point after these intensity-dependent effects have already taken place. So the additivity suggests that the cognitive act of covert orienting of visual attention operates fairly late in the visual detection process.

Early versus late enhancement can also be considered in anatomical terms. For example, "early" could correspond to the neuronal networks up to and including the primary visual cortex (area 17); "late" would then correspond to the extrastriate visual areas in the occipital, temporal, and parietal lobes. While this anatomical definition might be difficult to apply to 
higher order perceptual and cognitive tasks, it does provide a useful framework for discussing luminance detection. For example, Miller and Glickstein (1964, 1967) have shown that, in monkeys trained on a simple RT task, RTs to electrical stimulation of the primary visual cortex (area 17) are reduced relative to RTs to light stimuli by an amount equal to the latency of the visual evoked potential. The implications are that (1) the primary visual cortex is involved in the simple RT process, (2) it is probably involved as a serially organized component, and (3) the intensitydependent delays exert most of their impact on RT at or prior to the primary visual cortex. This is consistent with the fact that signal luminance strongly influences the response latency of photoreceptors (e.g., Baylor \& Hodgkin, 1973; Fuortes, 1958), ganglion cells (Cleland \& Enroth-Cugell, 1970; Lennie, 1981; Levick, 1973) and the visual cortex (Miller \& Glickstein, 1967; Vaughan, Costa, \& Gilden, 1966).

If attentional enhancement operated at these lower levels of the visual pathways, one might reasonably expect an interaction with signal luminance. This is because the responses of all of the neurons within this pathway saturate at high signal intensities, and any enhancement would be increasingly less advantageous as signal strength increased. Thus, although reticular inputs to the visual cortex (cf. Hughes, 1980) and lateral geniculate nucleus (Hughes \& Mullikin, 1984) could provide a potential pathway for early enhancement, the data provide no support for this hypothesis. Interestingly, these reticular effects on the visual pathway are not really selective; they produce a general increase in the excitability of visual cells, and therefore not only enhance the responses to visual inputs, but increase noise levels as well (in the form of increased spontaneous activity; cf. Singer, 1977; Singer, Tretter, \& Cynader, 1976). These considerations indicate that the neural structure most commonly associated with attention is probably not well suited to mediate this form of attention. Of course, other mechanisms for early enhancement might be suggested. However, it is probably worth emphasizing that many psychophysical experiments indicate that our sensory systems are nearly ideal receivers (Green, 1976; Hecht, Schlaer, \& Pirenne, 1942; Sivian \& White, 1933), and this suggests caution in attributing attentionally mediated facilitation of reaction times in terms of enhancing early sensory processing.

If, as the data of Miller and Glickstein suggest, effects that occur subsequent to the arrival of inputs to the visual cortex are additive with luminancedependent change in visual latency, then the effects of attention may also occur after area 17 , since signal luminance is essentially additive with attention facilitation. These considerations are in agreement with the recent report by Posner, Friedrich, Walker, and
Rafal (1983) that damage to the parietal lobes disrupts directed visual attention.

Finally, the present results need to be considered in light of the report by Bashinski and Bacharach (1980) that, in a signal detection experiment, attention was found to enhance sensitivity without affecting the response criterion. Probably the most significant difference between the present experiments and those of Bashinski and Bacharach (1980) lies in the strength of the signal. All of the luminances used in the present experiments were clearly suprathreshold, and therefore cannot be examined in the context of signal detection theory. Perhaps it is possible that attention might serve to enhance early visual processing, but that the use of stronger signals and/or the use of RT as a dependent measure is insensitive to this enhancement. It also seems possible, however, that SDT measures of sensitivity involve processes that include more than the earliest neural responses to signals. Specifically, to what depth of neural processing does a signal have to go before detection can be said to have occurred? As yet, there is, of course, no answer to this question, but it serves to emphasize the possibility that there may be processes within the visual system that occur subsequent to luminance-dependent effects, but nonetheless influence SDT measurements of sensitivity. In this context, there may be no inconsistency between the present results and those of Bashinski and Bacharach (1980). Perhaps future RT experiments using near-threshold luminances would clarify this issue. In any case, it is clear that attentional effects can easily be found at suprathreshold intensities, and that, under these conditions, directed attention and signal luminance exert primarily additive effects.

\section{EXPERIMENT 2}

As a cognitive process (one that depends on knowledge of the meaning of the precue), the act of orienting visual attention should be sensitive to the information value of the precue. Thus, the a priori probability that the precue is valid should influence the costs and benefits of attending to a spatial location. Although such effects have been shown using other paradigms, such as letter identification (Jonides, 1980), this variable has not been explored in a simple detection experiment on visual attention. The second experiment therefore explored the effect of the accuracy of information provided by the precue on simple RTs.

\section{Method}

Stimuli and Procedures. The stimuli and procedures were the same as in the first experiment, with the exception that the percentage of valid trials was 50,70 , or 90 . In addition, two different signal intensities were used (6.0 and $0.06 \mathrm{~m} \mathrm{fL})$, corresponding to 
the first and third intensities from Experiment 1. Each subject was tested with every combination of flash intensity and cue probability in separate sessions of $\mathbf{3 3 2}$ trials. Cue probability varied across subjects according to a Latin square, and the two intensities were counterbalanced across subjects. The subjects were always informed about the accuracy of the precue used in each session. Each subject participated in seven sessions (one practice run followed by six experimental sessions).

Subjects. Twelve introductory psychology students served as subjects. As before, all claimed to be emmetropic or optically corrected.

\section{Results}

Anticipatory error rates. Anticipations were excluded from the RT analysis and were analyzed separately in a four-factor analysis of variance (intensity $x$ cue validity $x$ cue type $x$ subjects). The results of this analysis indicated a significant main effect of cue type $[\mathrm{F}(2,22)=18.39, \mathrm{p}<.001]$ and a significant interaction between cue validity and cue type $[F(4,44)=$ $3.13, \mathrm{p}<.03]$. The mean error rate for valid trials was $5.9 \%$, the rate for invalid trials was $5.8 \%$, and that for neutral trials, $3.5 \%$. Post hoc comparisons revealed that neutral trials generated significantly fewer anticipations than either valid or invalid trials $(p<.05)$. An analysis of the cue type $x$ cue validity interaction suggested that the trend for lower anticipation rates on neutral trials was restricted to the $50 \%$ and $70 \%$ validity conditions; no differences were found when the cue validity was $90 \%$.

Reaction time data. The main results of the experiment are illustrated in Figure 3, which shows the mean reaction times for valid, invalid, and neutral trials for each of the three probability conditions at each of two signal intensities. An analysis of variance on these data indicated that intensity $[\mathrm{F}(1,11)=276.2, \mathrm{p}<.001]$ and cue type (valid, invalid, neutral) $[F(2,22)=45.2$, $p<.001]$ had significant effects; the main effect of probability (cue accuracy) was not significant $[F(2,22)$

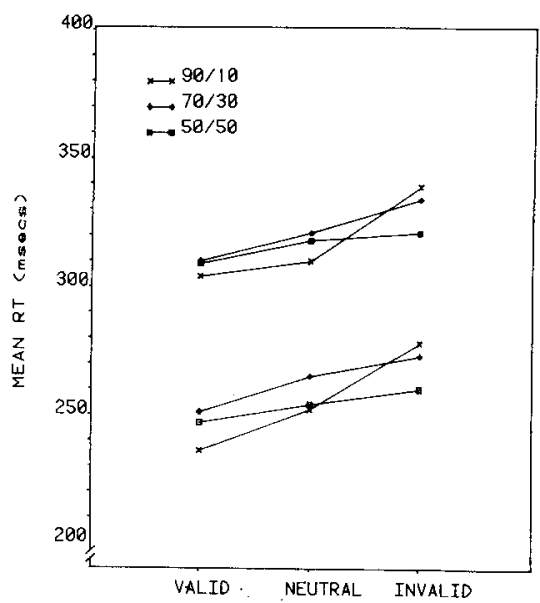

Figure 3. Effects of variations in the information value of the spatial precue on RTs for valid, neutral, and invalid trials at two different flash luminances. Cue validities are indicated in the insert.
$<1.0]$. There was no interaction between intensity and any of the other factors, but there was a significant interaction between cue type and cue probability $[F(4,44)=16.9, p<.001]$, indicating that the costs of invalid cues and the benefits of valid cues depend on the quality of information provided by the precue. Post hoc analysis of the interaction between cuing validity and cue type (valid, neutral, and invalid) indicated that valid, neutral, and invalid RTs were all significantly different from each other when the cuing validity was $90 \%$. When the cuing validity was $70 \%$, only the comparison between valid and neutral RTs failed to reach significance. When cuing validity was set at $50 \%$, only the invalid-neutral comparison failed to reach significance. Although it may seem odd that the data showed a significant attention effect (valid-invalid effect) when the cue validity was $50 \%$, it is important to bear in mind that all of the subjects were tested under all validity conditions, and, because of the design, most had received experience with valid precues $(70 \%, 90 \%$, or both) prior to their exposure to the $50 \%$ condition. Under these circumstances, it is probably difficult to treat the uninformative precue as truly neutral. This suggestion is supported by the fact that the cuing effect in the $50 \%$ condition was smaller in those subjects who received that condition first (Table 1).

I also compared valid RTs under each of the three validity conditions. These comparisons indicated that valid RTs under $50 \%$ and $70 \%$ validity did not differ, but all other comparisons among the valid RTs were. Similar comparisons for the invalid RTs showed no difference between the $70 \%$ and $90 \%$ conditions, although all other pairwise comparisons were significant. Thus, the principal difference between the $50 \%$ and $70 \%$ conditions was found on invalid RTs, while the valid RTs distinguished the $70 \%$ and $90 \%$ conditions.

As the $50 \%$ validity condition is informationally the same as providing the subjects with neutral cues, an interaction between cue type and cue probability is almost assured when the comparisons include the $50 \%$ condition. It is therefore important to establish whether costs and benefits vary between the $70 \%$ and $90 \%$ valid conditions, since both of these conditions do provide information useful for directing attention. Analysis of variance on the data from the .7 and .9 probability conditions yielded the same result; the probability $x$ cuing interaction was still highly significant $[F(2,22)$ $=17.04, p<.001]$. Thus, the attention effect clearly diminished with reductions in the validity of the cue.

In view of the finding that the strength of a subject's position expectancy influences the costs and benefits of directed visual attention, I explored the possibility that there may be momentary changes in expectancy resulting from the sequence of cues (valid, neutral, or invalid) presented on preceding trials. Such transient changes in expectancy might be re- 
Table 1

Mean Difference Scores (Invalid Mean/Valid Mean, Expressed in Milliseconds) for Subjects Who Either Did $(n=4)$ or Did Not $(n=8)$ Receive the $50 \%$ Validity Condition First

\begin{tabular}{lcc}
\hline & \multicolumn{2}{c}{ Flash Intensity } \\
\cline { 2 - 3 } & Dim & Bright \\
\hline $50 \%$ first & 6.0 & 3.5 \\
$70 \%$ or $90 \%$ first & 16.25 & 15.75 \\
\hline
\end{tabular}

vealed as sequence effects. For example, if in a $90 \%$ valid condition, a subject happens to get two invalid trials in succession, it might be supposed that he or she would expect the next trial to be valid. In the event that the next trial was invalid, one might expect the RTs to be slower than on an invalid trial following 5,6 , or 7 valid trials. All the data were sorted according to the sequence of valid and invalid trials (neutral trials were ignored) to a depth of seven trials. An analysis of variance of the means for each of these sequences revealed no significant sequence effects for any of the three probability conditions.

\section{GENERAL DISCUSSION}

The results from these experiments clearly indicate that the information value of the precue interacts with the effects of visual attention, whereas changes in signal strength exert simple additive effects. Thus, the attentional mechanism is sensitive to the strength of the subject's positional expectancy, but is not altered by the strength of the sensory signal itself. Although it might be argued that the range of luminance values used in this experiment was insufficient to detect an interaction (also see discussion of Experiment 1), it can be pointed out that the luminance manipulation had a much greater effect on RT than did changes in cue validity, and although the data show no evidence of an interaction with luminance, cue validity exerted strong interactive effects. Thus, luminance is additive over a range of RTs that far exceeds the range of RTs produced by variations in cue validity, but only cue validity produced interactive effects.

In general, increases in cue validity appear to speed RTs on valid trials and to slow RTs on invalid trials; in both luminance conditions, the slowest invalid RTs occurred when the cue validity was $90 \%$, and this condition also produced the fastest valid RTs. The picture is less clear when the $70 \%$ and $50 \%$ validity conditions are compared. In this case, the valid RTs are similar, but the $70 \%$ validity produced slower responding on the invalid trials. The principal difference between the $70 \%$ and $50 \%$ conditions therefore appears attributable to the costs associated with invalid cues. In fact, these data typically do show greater costs than benefits, which may help ex- plain why the costs more readily distinguish between the .5 and .7 validity conditions. The work by Posner and his colleagues (Posner, 1978; Posner et al., 1980) has tended to show symmetric costs and benefits, but there are exceptions (e.g., Figure 7.10 in Posner, 1978). I have no ready explanation for the modest deviations from symmetry in the present data. There are some stimulus differences between these experiments and those of Posner and his colleagues (e.g., we did not use two boxes to indicate the two possible target locations, and we used $100-\mathrm{msec}$ flashes instead of response-terminated flashes), but it is not clear why these differences should matter. Reaction times on neutral trials were always intermediate relative to valid and invalid trials, however, and could be statistically distinguished from valid RTs almost as often as invalid RTs. We now turn to a consideration of what these results mean in the context of current views of directed visual attention.

Posner et al. (1980) suggested that attention must be aligned with the source of input before detection responses can be initiated. According to this view, the differences in RTs on valid, neutral, and invalid trials result from the necessity of moving attention to the source of input, a process that takes time. Invalid trials are slowest because attention must move from the expected to the unexpected location (a distance of $8 \mathrm{deg}$ in the present experiments). The assumption is that when subjects receive a neutral cue, their attention remains at the fovea until the flash occurs, so attention need move only half as far on neutral trials as on invalid trials. Of course, movements of attention are not required on valid trials, since the mechanism is already aligned with the input. The important point here is that the differences in RTs on valid and invalid trials are assumed to reflect the time it takes to redirect attention from one location to another after the flash has already occurred. However, the finding that cue probability changes the size of the costs and benefits seems to present difficulties to this view, since it is not clear why the rate of movement should vary with cue validity.

There are at least three ways in which the effect of the strength of an observer's expectancy can be reconciled with the idea that postflash movements of visual attention must precede detection responses. First, one could argue that subjects commit their attention to the cued location less often as cue accuracy is reduced. Let us call this suggestion the "probability matching" hypothesis. According to this hypothesis, one would expect that the variance of the valid RT distribution should increase with decreasing cue validity; under probability matching the distribution reflects a mix of valid and neutral trials, and as validity is reduced the distribution includes an increasing proportion of trials in which attention has not been moved to the cued location. Similar arguments have been presented with respect to the same issue in a 
letter-recognition task (Jonides, 1980). This prediction was tested by comparing the standard deviations of the valid RT distributions in each of the three probability conditions. The data are illustrated in Figure 4. Analysis of variance yielded no significant differences, so the data provide no support for the probability matching hypothesis. Jonides (1980) reported similar findings using a letter-recognition task.

Alternatively, subjects could position their attention at a location somewhere between the cued location and the fixation mark. If the distance between the attentional focus and the cued location grew with decreased cue validity, then decreases in both the costs and benefits would accompany decreases in cue validity. Under this assumption, decreasing benefits with lower cue validity would be attributed to the small misalignment between attention and the cued location. As this presumed misalignment would leave the focus of attention closer to the uncued location, decreased costs could occur on invalid trials as well. We might call this suggestion the
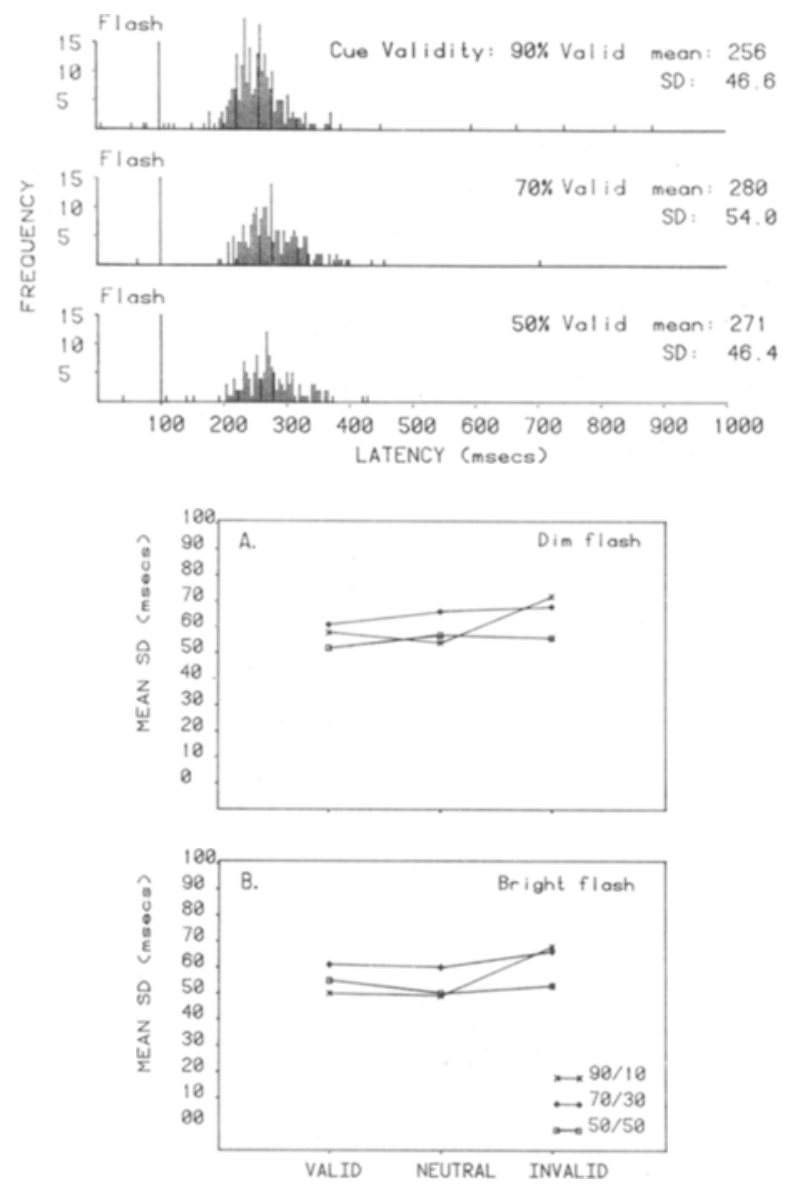

Figure 4. (A) Reaction time distributions obtained from one subject for valid trials under three different conditions of cue validity. (B) Means of the standard deviations of the valid, neutral, and invalid RT distributions for 12 subjects for two different flash luminances. "spatial matching" hypothesis. Information concerning the degree of facilitation of RTs for locations surrounding the cued location under conditions of low and high cue validity is needed to evaluate the merit of this idea.

A third possibility relates to the disengagement of attention from the cued location to the target (on invalid trials). A disengagement process logically should precede attentional movements, and disengagement has been suggested as the subprocess underlying the deficits shown by parietal lobe patients in this task (Posner et al., 1983). According to this view, the latency to disengage attention increases with increased cue validity. When the validity of the precue is high, subjects may take longer to redirect attention on invalid trials, not because the rate of movement is slower, but because the latency to begin the movement is increased. The present data do not permit an evaluation of this idea, and it would appear a difficult problem to approach experimentally.

It would appear, then, that there are a number of ways in which the present results could be explained within the context of a focal attentional mechanism that must be aligned with input signals prior to overt detection responses. There is, however, an important aspect of this attention effect that seems in direct conflict with this idea, indicating that other conceptualizations of the effect should be explored. Posner (1978) has shown that the size of the costs and benefits of directed visual attention are independent of eccentricity. This result is clearly incompatible with the suggestion of postflash attentional movements, and probably represents the strongest argument against the idea that the slowing of RTs on invalid trials is due to postflash movements of attention. Moreover, the failure to find a benefit of sequential over simultaneous presentations of letters in recognition tasks (Eriksen \& Spencer, 1969; Shiffrin \& Gardner, 1972; Shiffrin et al., 1973) is also incompatible with the idea that attention must be directed to a particular location before detection and recognition of the input can occur.

\section{Attention as an Analog Process}

The influence of the strength of an observer's expectancy on detection latency is consistent with an analog view of spatial attention in which the strength of the attentional process varies with the strength of expectancy. This suggestion is in accord with recent reports of analog-like attentional effects using other paradigms (e.g., Jonides, 1980; Shaw, Mulligan, \& Stone, 1983; Shaw \& Shaw, 1977). However, under the assumption that the mechanism has a limited spatial extent, we are still faced with the problem that the effect is independent of eccentricity. Rather than suggesting that the same mechanism produces both costs and benefits (i.e., attentional movements), an alternative approach might be to suggest that two 
distinct processes are at work in this situation, a facilitation of responses to signals in expected locations and an inhibition of responses to signals in unexpected locations. This idea has the attraction that the costs and benefits need not show any dependency with eccentricity.

\section{Summary}

These experiments clearly demonstrate that the effects of spatial attention are additive with signal strength and interactive with the strength of the subject's expectancies. These results must be incorporated into any coherent account of the manner in which expectancy influences detection latency, and some possible implications have been explored. While the additive effects of intensity are easily accounted for by suggesting that attention operates fairly late in the detection process (that is, after intensity-dependent coding processes), the effects of cue validity are more problematic. Although the data indicate that the strength expectancy and these manifestations of attention are closely related, additional experiments are needed to clarify the manner in which expectancy influences the detection process.

\section{REFERENCES}

Bashinski, H. S., \& Bacharach, V. R. (1980). Enhancement of perceptual sensitivity as the result of selectively attending to spatial locations. Perception \& Psychophysics, 28, 241-249.

Baylor, D. A., \& Hodakin, A. L. (1973). Detection and resolution of visual stimuli by turtle photoreceptors. Journal of Physiology (London), 234, 163-198.

Cleland, B. G., \& Enroth-Cugelt, C. (1970). Quantitative aspects of gain and latency in the cat retina. Journal of Physiology (London), 206, 73-91.

Colegate, R. L., Hoffman, J. E., \& Eriksen, C. W. (1973). Selective encoding from multielement visual displays. Perception \& Psychophysics, 14, 217-224.

Eriksen, C. W., \& Collins, J. F. (1969). Temporal course of selective attention. Journal of Experimental Psychology, 80, 254-261.

Eriksen, C. W., \& Hofrman, J. E. (1972). Temporal and spatial characteristics of selective encoding from visual displays. Perception \& Psychophysics, 12, 201-204.

Eriksen, C. W., \& Hofrman, J. E. (1973). The extent of processing of noise elements during selective encoding from visual displays. Perception \& Psychophysics, 14, 155-160.

Eriksen, C. W., \& Hoffman, J. E. (1974). Selective attention: Noise suppression or signal enhancement? Bulletin of the Psychonomic Society, 4, 587-589.

Eriksen, C. W., \& Spencer, T. J. (1969). Rate of information processing in visual perception: Some results and methodological considerations. Journal of Experimental Psychology, 79, 2, part 2.

Fúntes, M. G. F. (1958). Electrical activity of cells in the eye of the limulus. American Journal of Ophthalmology, 46, 210-223.

GreEN, D. M. (1976). Absolute sensitivity. In An introduction to hearing. Hillsdale, NJ: Erlbaum.

Hecht, S., Schlaer, S., \& Pihenne, M. H. (1942). Energy, quanta and vision. Journal of General Physiology, 25, 819-840.

HuGHES, H. C. (1980). Efferent organization of cat pulvinar complex, with a note on bilateral claustrocortical and reticulocor- tical connections. Journal of Comparative Neurology, 193 , 937-963.

Hughes, H. C., \& Kelsey, J. V. (1984). Response-dependent effects on near-threshold detection performance: Saccades versus manual responses. Perception \& Psychophysics, 35, 543-546.

Hughes, H. C., \& Mullikin, W. H. (1984). Brainstem afferents to the lateral geniculate nucleus of the cat. Experimental Brain Research, 54, 253-258.

Jonides, J. (1980). Towards a model of the mind's eye's movement. Canadian Journal of Psychology, 34, 103-112.

LenNie, P. (1981). The physiological basis of variations in visual latency. Vision Research, 21, 815-824.

Levick, W. R. (1973). Variation in the response latency of cat retinal ganglion cells. Vision Research, 13, 837-853.

Mille R, J. M., \& Glickstein, M. (1964). Reaction time to cortical stimulation. Science, 146, 1594-1596.

Miller, J. M., \& Glickste in, M. (1967). Neural circuits involved in visuomotor reaction time in monkeys. Journal of Neurophysiology, 30, 399-414.

Posne r, M. I. (1978). Orienting. In Chronometric explorations of mind (pp. 185-215). Hillsdale, NJ: Erlbaum.

POSNER, M. I. (1980). Orienting of attention. Quarterly Journal of Experimental Psychology, 32, 3-25.

Posner, M. I., Friedrich, F. J., Walker, J., \& Rafal, R. D. (1983). Neural control of covert visual orienting. Paper presented at the 24th Annual Meeting of The Psychonomic Society, San Diego.

Posnter, M. I., Snyder, C. R. R., \& Davidson, B. J. (1980). Attention and the detection of signals. Journal of Experimental Psychology: General, 109, 160-174.

Shaw, M. L., Mulligan, R. M., \& Stone, L. D. (1983). A test between two-state and continuous-state attention models. Perception \& Psychophysics, 33, 338-355.

Shaw, M. L., \& SHAw, P. (1977). Optimal allocation of cognitive resources to spatial locations. Journal of Experimental Psychology: Human Perception and Performance, 3, 201-211.

Shiffrin, R. M., \& Gardner, G. T. (1972). Visual processing capacity and attention control. Journal of Experimental Psychology, 93, 72-82.

Shiffrin, R. M., Gardner, G. T., \& Allmeyer, D. H. (1973). On the degree of attention and capacity limitations in visual processing. Perception \& Psychophysics, 14, 231-236.

Shulman, G. L., Remington, R. W., \& McLean, J. P. (1979). Moving attention through space. Journal of Experimental Psychology: Human Perception and Performance, 5, 522-526.

Singer, W. (1977). Control of thalamic transmission by corticofugal and ascending reticular pathways in the visual system. Physiological Reviews, 57, 386-420.

Singer, W., Tretter, F., \& Cynader, M. (1976). The effect of reticular stimulation on spontaneous and evoked activity in the cat visual cortex. Brain Research, 102, 71-90.

Sivian, L. J., \& White, S. D. (1933). On minimum audible sound fields. Journal of the Acoustical Society of America, 4, 288-371.

Skelton, J. M., \& Eriksen, C. W. (1976). Spatial characteristics of selective attention in letter matching. Bulletin of the Psychonomic Society, 1, 136-138.

Sterngerg, S. (1969). The discovery of processing stages: Extensions of Donder's method. In W. G. Koster (Ed.), Attention and performance II (pp. 276-315). Amsterdam: North Holland.

TsAL, Y. (1983). Movements of attention across the visual field. Journal of Experimental Psychology: Human Perception and Performance, 9, 523-530.

Vaughan, H. G., Costa, L. D., \& Gilden, L. (1966). The functional relation of visual evoked response and reaction time to stimulus intensity. Vision Research, 6, 645-656.

(Manuscript received January 31, 1984; revision accepted for publication June 6,1984 .) 